\title{
Expression of OX40 Gene and its Serum Levels in Neuromyelitis Optica Patients
}

https://doi.org/10.1515/bmc-2019-0007

received October 6, 2018; accepted December 21, 2018.

Abstract: Neuromyelitis optica (NMO), also known as Devic's disease, is an autoimmune disorder of the central nervous system (CNS) in which immune system cells and antibodies primarily attack the optic nerves and the spinal cord. OX 40 (CD134) is a tumornecrosis factor(TNF)-receptor family member expressed primarily on activated $\mathrm{CD}^{+}$and $\mathrm{CD}^{+} \mathrm{T}$-cells. In an autoimmune disease, OX40 is typically up-regulated at sites of inflammation, and increases in the number of peripheral CD4 ${ }^{+} \mathrm{T}$-cells expressing OX40. OX40 and its ligand OX40L are key TNF members that augment T-cell expansion, cytokine production, and promote T-cell survival. The aim of this study was to evaluate and compare of $O X 40$ gene expression and its serum levels in patients with NMO and healthy controls. Twenty sex-/agematched healthy controls $(\mathrm{HC})$ (median age $=32$ years, 15 females/5 males) were engaged for the present study. Expression of $O X 40$ at the transcript level and serum protein levels were measured by quantitative real-time polymerase chain reaction (qRT-PCR) and enzyme-linked immunosorbent assays, respectively. The results indicated OX40 expression in patients was significantly lower than in healthy controls $(\mathrm{p}=0.001)$. However, the serum level of OX40 was not significantly different between groups $(\mathrm{p}=0.37)$. In addition, the results indicated that CD134

\footnotetext{
*Corresponding author: Nahid Eskandari, Department of Immunology, Faculty of Medicine, Isfahan University of Medical Sciences, Isfahan, Iran; Department of Physiology, Applied Physiology Research Center, Isfahan University of Medical Sciences, Isfahan, Iran, E-mail: neskandari@med.mui.ac.ir Parya Alidadiani: Department of Immunology, Faculty of Medicine, Isfahan University of Medical Sciences, Isfahan, Iran Arezou Dabiri: Department of Immunology, International Campus, Shahid Sadoughi University of Medical Sciences, Yazd, Iran Vahid Shaygannejad, Omid Mirmosayyeb: Isfahan Neuroscience Research Center, Department of Neurology, Alzahra Hospital, Isfahan University of Medical Sciences, Isfahan, Iran

Hamidreza Jahanbani-Ardakani: Isfahan Medical Students Research Center (IMSRC), Isfahan University of Medical Sciences, Isfahan, Iran Mostafa Manian: Department of Immunology, School of Medicine, Iran University of Medical Sciences, Tehran, Iran
}

expression was not age-related $(\mathrm{p}=0.041)$. Overall, this study suggests to us that OX40 levels are not a suitable marker for diagnosis or treatment of NMO.

Keywords: Neuromyelitis optica (NMO); OX40; autoimmune.

\section{Introduction}

Neuromyelitis optica (NMO), also known as Devic's disease, is an autoimmune disorder of the central nervous system (CNS) in which immune system cells and antibodies attack the optic nerves and the spinal cord. Like multiple sclerosis (MS), NMO is a relapsing-remitting disease $[1,2]$. During any relapse, new damage to the optic nerves and/or spinal cord can lead to accumulating disability. Unlike MS, there is no progressive phase of this disease. Therefore, preventing attacks is critical to a good long-term outcome.

Among NMO and NMO spectrum disorder (NMOSD) patients, $>70 \%$ are characterized by a presence of serum autoantibodies against astrocyte water channel aquaporin 4 (AQP4) on cell membranes [3, 4]. These pathogenic IgG antibodies are important as they illustrate how AQP4specific T-cells likely participate in the pathogenesis of NMO [5]. Among these, there is an IgG $_{1}$ autoantibody that is also found in patients with two related neurologic conditions (two-sided optic neuritis (ON) and longitudinal wide transverse myelitis (LETM)) that fall in the NMO range of diseases, [5]. Thus, there is the potential to develop regimens to counteract these and other antibodies once the latter have been identified - as a means to help prevent/mitigate NMO attacks.

OX40 (CD134) is a tumor necrosis factor (TNF)-receptor family member expressed primarily on activated $\mathrm{CD}^{+}$ and, $\mathrm{CD}^{+}$and regulatory T-cells ( $\mathrm{T}_{\text {reg }}$ cells) [6]. Normally, OX40 is activated by the ligand OX40L (CD134L), and thus functions as a T-cell co-stimulatory molecule. It is known that OX40-OX40L interactions can promote T-cell survival, including that of effector and memory T-cells, as 
Table 1: Characteristics of NMO patient and control subjects.

\begin{tabular}{lll}
\hline & NMO & Controls \\
\hline Females/Males & $15 / 5$ & $15 / 5$ \\
Average age at onset & 31 & 31 \\
AQ-P4 Ab positive (\%) & 30 & --- \\
LETM (\%) & 60 & --- \\
Normal brain (MRI) (\%) & 80 & --- \\
EDSS (n) & 2 & --- \\
\hline
\end{tabular}

Values are presented as frequencies (percentages)

LETM: Longitudinally-extensive transverse myelitis, annualized relapse rate.

EDSS: Expanded Disability Status Scale.

well as enhance the production of several cytokines [7-9]. Studies have shown that use of anti-OX40 in the treatment of some autoimmune diseases imparts a potent antiinflammatory activity in a manner that is dependent on both $\mathrm{CD}^{+}$and $\mathrm{CD} 8^{+} \mathrm{T}$-cells $[10,11]$. Immunization models have also shown that the use of anti-OX40 increases T-cell proliferation and differentiation into expanded populations of effector T-cells, with concomitant changes in cytokine production, T-cell-mediated cytotoxicity, and a decrease in activation-induced cell death [11, 12].

In several autoimmune diseases (e.g., Systemic lupus erythmathosus [SLE], colitis), the presence of OX40 is up-regulated at sites of inflammation, and there are increases in the number of peripheral $\mathrm{CD}^{+} \mathrm{T}$-cells expressing OX40 $[13,14]$. In SLE, the expression of OX40 on $\mathrm{CD}^{+} \mathrm{T}$-lymphocytes and the serum level of OX40L may act as markers of lupus nephritis and it suggests there is a correlation between the presence of OX40 expression and disease severity[15]. As OX40 also promotes the colitogenic T-helper cell type $1\left(\mathrm{~T}_{\mathrm{H}} 1\right)$ response, its expression on $\mathrm{T}_{\text {reg }}$ cells may be required for effective competition with OX40-dependent effector responses [14, 16]. In addition, stimulation of OX40-expressing cells after the induction of colitis exaggerates intestinal inflammation - presumably by enhancing effector lymphocyte function. Thus, it seems that stimulation of a host with an agonistic OX40 antibody could increase the number of functional $\mathrm{T}_{\text {reg }}$ cells that, in turn, can impact on any subsequent developing/ ongoing immune response $[13,16,17]$.

Soluble OX40 (sOX40) may be produced through cleavage from cell surface OX 40 and/or alternative splicing [18-20]. Soluble forms of TNF receptors have antagonistic effects on membrane-bound receptors. Therefore, it is possible that abnormal activation of T-cells may induce alternative splicing of sOX40 in patients with NMO [9, 12, 21]. However, the function of sOX40 in NMO remains unknown.

The OX40 expression level in peripheral white blood cells of NMO patients may provide insight into immunologic mechanisms related to NMO progression. Accordingly, this study was designed to evaluate OX40 gene expression and serum levels (as sOX40) of the protein for potential use in helping in the eventual development of more effective targeted treatments against this pathology.

\section{Materials and Methods}

\section{Patients and controls}

Twenty NMO patients were recruited for the study (median age $=31$ years, 15 female/5 male; mean disease duration $=2.4$ years) from the Kashani Hospital of Isfahan (Iran). Diagnosis of NMO was confirmed by clinical and neurophysiological parameters. All patients met research criteria used to define NMO [3], as verified by a hospital neurologist. For all patients, both the longitudinallyextensive transverse myelitis (LETM) as annualized relapse rate and the Expanded Disability Status Scale (EDSS) were assessed. Cerebral and spinal cord magnetic resonance imaging (MRI) were used to evaluate the presence of specific NMO-like lesions. As controls, 20 healthy sex- $/$ age-matched individuals $(\mathrm{HC}$; median age $=32$ years, 15 female/5 male) were also recruited for study. Table 1 presents the demographic and clinical characteristics of all study subjects.

A total of $5 \mathrm{ml}$ fasting venous blood was taken from each individual. Two ml was collected into EDTAcontaining tubes for subsequent use in white blood cell RNA extraction. The other $3 \mathrm{ml}$ was collected into a tube without anti-coagulant; this material was immediately centrifuged ( $\left.4000 \mathrm{rpm}, 6 \mathrm{~min}, 4^{\circ} \mathrm{C}\right)$, and the resultant serum was harvested and stored at $-20^{\circ} \mathrm{C}$ for later analysis.

Informed consent: Informed consent has been obtained from all individuals included in this study.

Ethical approval: The research related to human use has been complied with all the relevant national regulations, institutional policies and in accordance the tenets of the Helsinki Declaration, and has been approved by the ethical committee of Isfahan University of Medical Sciences. 


\section{RNA extraction and RT-PCR}

From each whole blood sample, total RNA was extracted from white blood cells using a YTA kit Total RNA Purification Mini kit(Yekta Tajhiz, Tehran, Iran), according to manufacturer's protocol. Concentration and integrity of total isolated RNA from each sample was assessed using a Nanophotometer Pipette System (Helmholtz, Nauenberg, Germany) and the material was then stored at $-70^{\circ} \mathrm{C}$ (mean concentration was $\approx 80 \mu \mathrm{g} / \mathrm{ml}$ ).

From each sample, cDNA was prepared using a Thermo Scientific Revert Aid RT Kit containing oligo dT primer mixture (Bio-Rad, Watford, UK) according to manufacturer protocol. In brief, $11 \mu \mathrm{l}(\approx 0.9 \mu \mathrm{g})$ total RNA was mixed with $1 \mu$ loligo dT primer, and then incubated at $65^{\circ} \mathrm{C}$ for $\left.5 \mathrm{~min}\right)$. Thereafter, $8 \mu \mathrm{l}$ Reverse Transcription Master Mix (5X Reaction Buffer) and 2X dNTP Mix (both Applied Biosystems, Cheshire, UK) was added (on ice), and the mixture was incubated for $1 \mathrm{hr}$ at $42^{\circ} \mathrm{C}$ and then $10 \mathrm{~min}$ at $70^{\circ} \mathrm{C}$.

\section{Real-time PCR}

qRT-PCR was performed using Syber Green PCR Master Mix (Applied Biosystems). Forward and reverse primer sequences used for the qRT-PCR reactions (Table 2) were designed using Allele ID version 7. Each qPCR was performed in a Step One Plus Real-Time PCR system (Applied Biosystems). Thermocycler conditions used in each case were $95^{\circ} \mathrm{C}$ for $15 \mathrm{~min}$ (initial denaturation) followed by 40 cycles of $95^{\circ} \mathrm{C}$ for $15 \mathrm{sec}, 54^{\circ} \mathrm{C}$ for $60 \mathrm{sec}$, and $72^{\circ} \mathrm{C}$ for $20 \mathrm{sec}$, with a final extension of $72^{\circ} \mathrm{C}$ for 1 min. All results were evaluated using the $2^{\text {DDCt }}$ method [22, 23].

\section{ELISA}

sOX40 levels in sera were measured (in duplicate) using a human sCD134 (OX40) platinum ELISA kit (eBioscience, Vienna, Austria), following manufacturer protocols. Optical density (OD) at $450 \mathrm{~nm}$ wavelength was recorded for each sample using a Hiperion Microplate Reader (Rodermark, Germany). To establish seropositivity, serum from each NMO patient was also evaluated for anti-Aquaporin 4 (AQP4) antibody using an Indirect Immunofluorescence Test (IIFT) cell-based assay (EUROIMMUN IIFT, Luebeck, Germany).
Table 2: Real-time quantitative PCR primers used in the study.

\begin{tabular}{ll}
\hline Primer & Sequence \\
\hline GAPDH Forward & AAGCTCATTTCCTGGTATG \\
GAPDH Reverse & CTTCCTCTTGTGCTCTTG \\
OX40 Forward & TGGTGTAACCCAGAAGTG \\
OX40 Reverse & GTCAACTCCAGGCTTGTA \\
\hline
\end{tabular}

\section{Statistical analysis}

All results are expressed as mean \pm SD. Normal distribution of all data was determined using a Kolmogorov-Smirnov test. A non-parametric Mann-Whitney $U$-test was applied for comparing values between groups. A p-value $<0.05$ was accepted as significant. All analyses were conducted using an SPSS 20 package (SPSS Inc., Chicago, IL).

\section{Results}

\section{OX40 mRNA expression}

OX40 mRNA in the white blood cells of NMO patients was found to be significantly $(p=0.001)$ lower than in control individuals' cells. The average Ct values were 32.1 and 29.7, respectively. Within-gender analyses revealed that females with NMO had levels significantly ( $p=0.041$ ) lower than in female control counterparts; average values were 32.8 and 30.4, respectively. There were no significant differences between male NMO and male controls $(\mathrm{p}=0.70)$. In total, there appeared to be no correlation between OX40 mRNA and age in this group of NMO patients.

\section{Serum s0X40 levels}

The ELISA data indicated there were no significant differences between sOX40 levels in the serum of NMO patients compared with those from healthy controls $(\mathrm{p}=$ 0.37) (Figure 1). The average values were 12.7 and $14.9 \mathrm{ng} /$ $\mathrm{ml}$, respectively. The current analyses found that median sOX40 levels in AQP4 seropositive subjects were higher than in seronegative NMO patients, but this difference was not significant $(\mathrm{p}=0.45)$ (Figure 2$)$. 


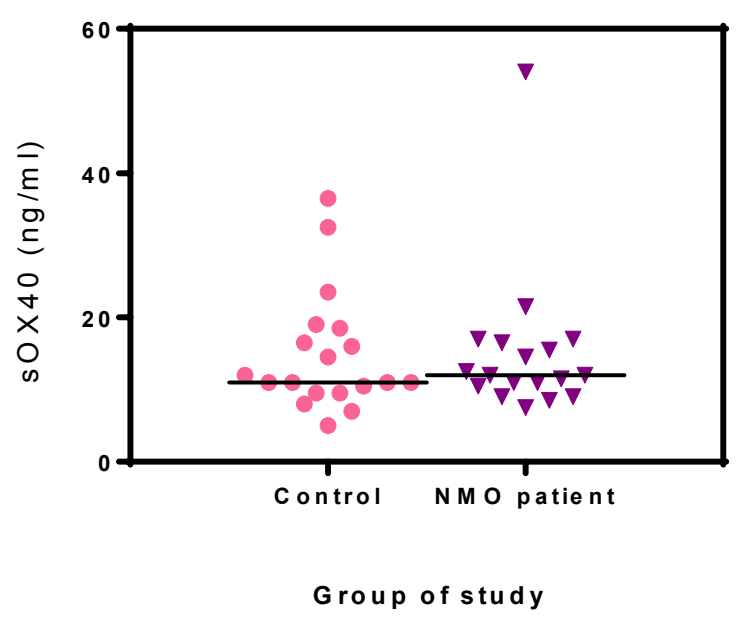

Figure 1: Serum s0X40 levels. There were no significant differences in SOX40 serum levels when comparing NMO patients with controls $(p=0.37)$. Values shown are means \pm SD of 20 subjects/group.

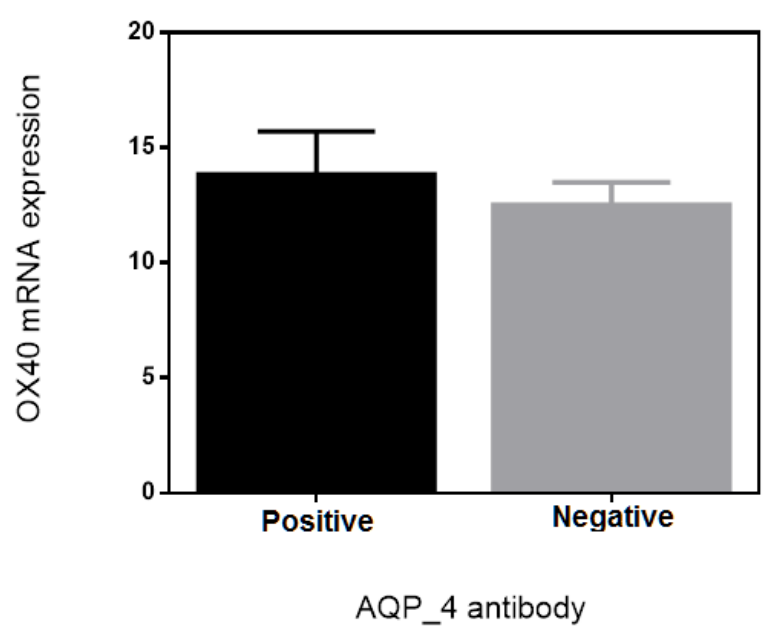

Figure 2: $\mathrm{SOX} 40$ serum levels in AQP4 seropositive and -negative NMO patients. There were no significant differences between the two groups $(p=0.044$.). Values shown are means \pm SD from 6 seropositive and 12 seronegative $\mathrm{NHO}$ patients.

\section{Discussion}

Several studies have provided evidence for an important role of effector T-cells in various steps during the pathogenesis of neuromyelitis optica (NMO). Levels of interleukin (IL)-17-secreting CD4 ${ }^{+} \mathrm{T}$-cells $\left(\mathrm{T}_{\mathrm{H}} 17\right.$ cells) are known to be significantly elevated in NMO patients, suggesting these autoantigen-specific T-cells might help drive some of the pathologies associated with NMO [24]. Interestingly, IL-6 is not only elevated in the cerebral-spinal fluid (CSF), but also in the sera, of patients with NMO. This is important in that IL-6 is involved in development of $\mathrm{T}_{\mathrm{H}} 17$ cells [25]. Serum IL-22 and IL-21 levels are also elevated during NMO [26] and so it is not surprising that $\mathrm{T}_{\mathrm{H}} 22$ cell levels have been found to significantly correlate with those of $\mathrm{T}_{\mathrm{H}} 17$ cells in these patients. Certain types of T-cells are also found in the lesions that occur in NMO patients, though their antigen specificity and function have not been characterized [27]. Thus, a variety of effector T-cells seem to be potentially required for overall NMO development. Whether these are specifically needed for disease-related lesion development, or for disrupting blood-brain or blood-CSF barriers, or just perhaps for creating an inflammatory milieu in situ for anti-AQP4 antibodies to be operational remains to be determined.

At the factor level, a variety of co-stimulatory molecules (such as CD69) have been shown to play important roles in the NMO pathogenesis. It is plausible to assume that the presence - or absence - of other factors may also likely contribute to T-cell activation in NMO [5]. OX40 is essential for optimal function of T-cells. For example, a role for OX40 signaling in activation of cytolytic T-lymphocytes is well established [14]. Expression of OX40 is also believed to be a factor involved in the ability of T-cells to leave the general circulation and enter the CNS [28]. In addition, OX40 has an important role in preventing T-cell apoptosis by inducing expression of anti-apoptotic molecules (such as Bcl-X). When taken together, it is clear that OX40 can impact on the size of the effector T-cell pool in a host. Accordingly, as critical components of T-cell responses, OX40 is likely to be important in the context of autoimmunity [29-31].

In this study, it was seen that gene expression of OX40 was significantly decreased in the peripheral white blood cells of NMO patients. At the same time, circulating levels of the soluble form of the OX40 protein (sOX40) was unaltered by disease status. This observation contrasts with that of another investigations wherein sOX40 levels in sera of patients with ALS were decreased compared to control values [32]. Other reports have shown that active human NMO lesions contained increased numbers of $\mathrm{CD}^{+} \mathrm{T}$-cells expressing the OX40 activation marker, and that expression was more profound compared to that seen in MS lesions of comparable activity [33]. Recently, it was shown that T-cells isolated from the spinal cords of Lewis rats with actively-induced EAE (experimental allergic encephalomyelitis) expressed OX40 [28]. As such, it is not clear why systemic levels of OX40 (specifically, as soluble sOX40 form) in NMO patients here were the same as in the control subjects. Similarly, it is not readily apparent why expression levels of OX40 gene among circulating leukocytes in the NMO patients here were decreased in comparison to in control subject cells. 
The present study indicated that OX40 gene expression in circulating white blood cells was significantly lower in NMO females than in cells of corresponding female controls. Interestingly, there was no similar male-related differential in outcome. While it is known sex hormones (including estrogens and androgens) have crucial roles in autoimmune diseases [34], it is not clear why there was such a bifurcation in outcomes between the male and female NMO patients (vs. controls) here.

Lastly, the present study showed OX40/CD134 expression (serum) was not age-related among the NMO patients. It is known that CD134 surface density on CD4 ${ }^{+}$ cells was decreased in spinal cords of aging rats and on in vitro-stimulated naïve $\mathrm{CD}^{+}$splenocytes taken from aged rats vs. cells from younger rats [35]. This invites the question of if expression of the marker is impacted by localization to a given tissue, as opposed to any similar influence on T-cells while they remain in the general circulation. This specific issue warrants further study.

Acknowledgments: This study was supported by the Isfahan University of Medical Sciences. The authors would like to thank to all employees of the Central Laboratory of IUMS for their help in the performance of this study.

Conflict of interest: Authors state no conflict of interest.

Declaration: The authors alone are responsible for the content of this manuscript.

\section{References}

1. Jacob A, McKeon A, Nakashima I, Sato DK, Elsone L, Fujihara $\mathrm{K}$, et al. Current concept of neuromyelitis optica (NMO) and NMO spectrum disorders. J Neurol Neurosurg Psychiatry. 2013;84(8):922-30.

2. Matiello M, Jacob A, Wingerchuk DM, Weinshenker BG. Neuromyelitis optica. Current opinion in neurology. 2007;20(3):255-60.

3. Morrow MJ, Wingerchuk D. Neuromyelitis optica. Journal of Neuro-Ophthalmology. 2012;32(2):154-66.

4. Trebst C, Jarius S, Berthele A, Paul F, Schippling S, Wildemann B, et al. Update on the diagnosis and treatment of neuromyelitis optica: recommendations of the Neuromyelitis Optica Study Group (NEMOS). Journal of neurology. 2014;261(1):1-16.

5. Drori T, Chapman J. Diagnosis and classification of neuromyelitis optica (Devic's syndrome). Autoimmunity reviews. 2014;13(4-5):531-3.

6. Akiba H, Oshima H, Takeda K, Atsuta M, Nakano H, Nakajima A, et al. CD28-independent costimulation of $T$ cells by
OX40 ligand and CD70 on activated B cells. The Journal of Immunology. 1999;162(12):7058-66.

7. Ishii N, Takahashi T, Soroosh P, Sugamura K. OX40OX40 ligand interaction in T-cell-mediated immunity and immunopathology. Advances in immunology. 105: Elsevier; 2010. p. 63-98.

8. Kaur D, Brightling C. OX40/0X40 ligand interactions in T-cell regulation and asthma. Chest. 2012;141(2):494-9.

9. Webb GJ, Hirschfield GM, Lane PJ. OX40, OX40L and autoimmunity: a comprehensive review. Clinical reviews in allergy \& immunology. 2016;50(3):312-32.

10. Sand IK. Neuromyelitis optica spectrum disorders. CONTINUUM: Lifelong Learning in Neurology. 2016;22(3, Multiple Sclerosis and Other Demyelinating Diseases):864-96.

11. Sato DK, Lana-Peixoto MA, Fujihara K, de Seze J. Clinical spectrum and treatment of neuromyelitis optica spectrum disorders: evolution and current status. Brain Pathology. 2013;23(6):647-60.

12. Linch SN, McNamara MJ, Redmond WL. OX40 agonists and combination immunotherapy: putting the pedal to the metal. Frontiers in oncology. 2015;5:34.

13. Griseri T, Asquith M, Thompson C, Powrie F. OX40 is required for regulatory T cell-mediated control of colitis. Journal of Experimental Medicine. 2010;207(4):699-709.

14. Sitrin J, Suto E, Wuster A, Eastham-Anderson J, Kim JM, Austin CD, et al. The 0x40/0x40 Ligand Pathway Promotes Pathogenic Th Cell Responses, Plasmablast Accumulation, and Lupus Nephritis in NZB/W F1 Mice. Journal of immunology (Baltimore, Md : 1950). 2017;199(4):1238-49.

15. Patschan S, Dolff S, Kribben A, Dürig J, Patschan D, Wilde B, et al. CD134 expression on CD4+ T cells is associated with nephritis and disease activity in patients with systemic lupus erythematosus. Clinical \& Experimental Immunology. 2006;145(2):235-42.

16. Zander RA, Obeng-Adjei N, Guthmiller JJ, Kulu DI, Li J, Ongoiba A, et al. PD-1 co-inhibitory and OX40 co-stimulatory crosstalk regulates helper $T$ cell differentiation and anti-Plasmodium humoral immunity. Cell host \& microbe. 2015;17(5):628-41.

17. Kroemer A, Xiao X, Vu MD, Gao W, Minamimura K, Chen M, et al. OX40 controls functionally different $T$ cell subsets and their resistance to depletion therapy. The Journal of Immunology. 2007;179(8):5584-91.

18. Compaan DM, Hymowitz SG. The crystal structure of the costimulatory $0 \times 40-0 \times 40 \mathrm{~L}$ complex. Structure. 2006;14(8):1321-30.

19. Jenkins SJ, Perona-Wright G, Worsley AG, Ishii N, MacDonald AS. Dendritic cell expression of OX40 ligand acts as a costimulatory, not polarizing, signal for optimal Th2 priming and memory induction in vivo. The Journal of Immunology. 2007;179(6):3515-23.

20. Lei W, Zeng D-X, Zhu C-H, Liu G-Q, Zhang X-Q, Wang C-G, et al. The upregulated expression of OX40/0X40L and their promotion of T cells proliferation in the murine model of asthma. Journal of thoracic disease. 2014;6(7):979.

21. Montler R, Bell RB, Thalhofer C, Leidner R, Feng Z, Fox BA, et al. OX40, PD-1 and CTLA-4 are selectively expressed on tumor-infiltrating $T$ cells in head and neck cancer. Clinical \& translational immunology. 2016;5(4):e70. 
22. Livak KJ, Schmittgen TD. Analysis of relative gene expression data using real-time quantitative PCR and the 2- $\triangle \Delta C T$ method. methods. 2001;25(4):402-8.

23. Wong ML, Medrano JF. Real-time PCR for mRNA quantitation. Biotechniques. 2005;39(1):75-85.

24. Blanc F, Noblet V, Jung B, Rousseau F, Renard F, Bourre B, et al. White matter atrophy and cognitive dysfunctions in neuromyelitis optica. PLoS One. 2012;7(4):e33878.

25. Kimura A, Kishimoto T. IL-6: regulator of Treg/Th17 balance. European journal of immunology. 2010;40(7):1830-5.

26. Wang H, Dai Y, Qiu W, Lu Z, Peng F, Wang Y, et al. Interleukin17-secreting $T$ cells in neuromyelitis optica and multiple sclerosis during relapse. Journal of Clinical Neuroscience. 2011;18(10):1313-7.

27. Lin J, Li X, Xia J. Th17 cells in neuromyelitis optica spectrum disorder: a review. The International journal of neuroscience. 2016;126(12):1051-60.

28. Carboni S, Aboul-Enein F, Waltzinger C, Killeen N, Lassmann H, Peña-Rossi C. CD134 plays a crucial role in the pathogenesis of EAE and is upregulated in the CNS of patients with multiple sclerosis. Journal of neuroimmunology. 2003;145(1-2):1-11.

29. Balashov KE, Rottman JB, Weiner HL, Hancock WW. CCR5+ and CXCR3+ T cells are increased in multiple sclerosis and their ligands MIP- $1 \alpha$ and IP-10 are expressed in demyelinating brain lesions. Proceedings of the National Academy of Sciences. 1999;96(12):6873-8.

30. Chen L, Flies DB. Molecular mechanisms of T cell co-stimulation and co-inhibition. Nature Reviews Immunology. 2013;13(4):227.

31. Gerondakis S, Siebenlist U. Roles of the NF-kB pathway in lymphocyte development and function. Cold Spring Harbor perspectives in biology. 2010;2(5):a000182.

32. Itżecka J. Serum soluble $0 \times 40$ in patients with amyotrophic lateral sclerosis. Acta Clinica Croatica. 2012;51(1):3-6.

33. Pohl M, Kawakami N, Kitic M, Bauer J, Martins R, Fischer M-T, et al. $T$ cell-activation in neuromyelitis optica lesions plays a role in their formation. Acta neuropathologica communications. 2013;1(1):85.

34. González DA, Díaz BB, Pérez MdCR, Hernández AG, Chico BND, de León AC. Sex hormones and autoimmunity. Immunology letters. 2010;133(1):6-13.

35. Djikić J, Nacka-Aleksić M, Pilipović I, Stojić-Vukanić Z, Bufan $B$, Kosec $D$, et al. Age-associated changes in rat immune system: lessons learned from experimental autoimmune encephalomyelitis. Experimental gerontology. 2014;58:179-97. 